\title{
Confinement-Enhanced Electron Transport across a Metal-Semiconductor Interface
}

\section{Citation}

Altfeder, I. B., J. A. Golovchenko, and V. Narayanamurti. 2001. “Confinement-Enhanced Electron Transport Across a Metal-Semiconductor Interface." Physical Review Letters 87 (5) (July). doi:10.1103/physrevlett.87.056801.

\section{Published Version}

doi:10.1103/PhysRevLett.87.056801

\section{Permanent link}

http://nrs.harvard.edu/urn-3:HUL.InstRepos:21976372

\section{Terms of Use}

This article was downloaded from Harvard University's DASH repository, and is made available under the terms and conditions applicable to Other Posted Material, as set forth at http:// nrs.harvard.edu/urn-3:HUL.InstRepos:dash.current.terms-of-use\#LAA

\section{Share Your Story}

The Harvard community has made this article openly available.

Please share how this access benefits you. Submit a story.

\section{Accessibility}




\title{
Confinement-Enhanced Electron Transport across a Metal-Semiconductor Interface
}

\author{
I. B. Altfeder, J. A. Golovchenko, and V. Narayanamurti \\ Division of Engineering and Applied Science, Harvard University, Cambridge, Massachusetts 02138 \\ and Department of Physics, Harvard University, Cambridge, Massachusetts 02138
}

(Received 26 December 2000; published 11 July 2001)

\begin{abstract}
We present a combined scanning tunneling microscopy and ballistic electron emission microscopy study of electron transport across an epitaxial $\mathrm{Pb} / \mathrm{Si}(111)$ interface. Experiments with a self-assembled $\mathrm{Pb}$ nanoscale wedge reveal the phenomenon of confinement-enhanced interfacial transport: a proportional increase of the electron injection rate into the semiconductor with the frequency of electron oscillations in the $\mathrm{Pb}$ quantum well.
\end{abstract}

DOI: $10.1103 /$ PhysRevLett.87.056801

The physical properties of self-organized epitaxial films are important for the study of low-dimensional electron transport [1,2], electron confinement in self-assembled nanostructures [3-5], and geometry of the buried interfaces [6,7]. These films often consist of an extremely thin epitaxial wetting layer uniformly covering a semiconductor surface, and three-dimensional self-assembled nanostructures that grow on the top of this layer by a mechanism known as the Stranski-Krastanov growth mode. Among the remarkable features of these films is a continuous metallic surface conductivity, which allows one to electrically contact individual nanostructures to study their transport properties. Understanding the influence of electron confinement, i.e., resonant electron states and size-dependent electron energy quantization, on the transport properties in these nanostructures is of fundamental importance in the development of nanoscale electronic devices. The experimental study of such quantum transport phenomena in nanostructures can be done with a combination of scanning tunneling microscopy (STM) [8] and ballistic electron emission microscopy (BEEM) [9-12].

Being a three-terminal modification of STM, BEEM is a powerful method for the study of transport phenomena. In the BEEM experiment, the semiconductor surface is usually covered with a thin $(\sim 50 \AA)$ metal layer, where the electrical potential is typically kept at zero. At low tip-sample bias, electrons tunneling from the STM tip spread through the metallic surface of the sample. However, when the energy of the tunneling electrons exceeds the Schottky barrier height, which is typically about $1 \mathrm{eV}$, some of these electrons are injected into the bulk of the semiconductor. The injected current is then collected by an Ohmic contact at the back side of the semiconductor substrate. BEEM methodology has been previously used in the study of Schottky barriers $[9,13]$, semiconductor density of states [14], resonant transport through semiconductor heterostructures [14] and buried quantum dots [15], and defects at buried interfaces [16].

In this Letter, we report the first combined STM/BEEM experiment on extremely thin metal islands that selfassemble during epitaxial growth. The results show a
PACS numbers: 73.21.-b, 68.37.Ef, 73.40.-c, 81.07.-b

proportional relationship between the ballistic transport across the epitaxial interface and the electron confinement energy in a metallic quantum well (QW), suggesting a new model for interfacial transport in this regime.

It has been previously shown that, on the $\mathrm{Si}(111)$ substrate, $\mathrm{Pb}$ islands begin to grow after an initial continuous double layer of $\mathrm{Pb}$ wets the surface [17]. On stepped substrates, $\mathrm{Pb}$ islands often possess a wedge-shaped geometry with an atomically flat top [4]. In this geometry, the metal thickness varies in a discrete manner due to atomic steps at the substrate-metal interface. Using these wedge-shaped nanostructures in conjunction with STM allows a convenient in situ adjustment of the metallic QW thickness. These properties make the $\mathrm{Pb} / \mathrm{Si}$ nanostructures a perfect object for the study of both electron confinement effects and quantum transport phenomena.

The semiconductor substrate for our experiment was cut from a lightly doped $n$-type $\mathrm{Si}(111)$ wafer. An Ohmic back contact was fabricated ex situ by As implantation, which created a heavily doped $n$-type layer. The sample was placed into an ultrahigh vacuum (UHV) chamber with a base pressure of $1.5 \times 10^{-10}$ Torr, where its surface was cleaned by a sequence of direct current heating $\left(1100{ }^{\circ} \mathrm{C}\right)$ and ion-beam sputtering $\left(\mathrm{Ne}^{+}\right)$. The quality and the chemical composition of the surface were monitored by Auger electron spectroscopy and low-energy electron diffraction. Following the cleaning, $\mathrm{Pb}$ atoms were deposited from an effusion cell onto a $7 \times 7$ reconstructed $\mathrm{Si}(111)$ substrate. The sample was then in situ transferred to a UHV STM operating at room temperature.

The schematic of the experiment is presented in Fig. 1. The electrical contact to the surface $\mathrm{Pb}$ overlayer was made in situ using a spring contact located $1 \mathrm{~mm}$ apart from the STM tip. This contact was grounded to provide a separate conductance path for low-energy electrons. The current injected into the substrate, $I_{c}$, was measured with a picoammeter connected to the back side Ohmic contact held at zero potential. During the collector current vs tunnel bias $\left(I_{c}-V\right)$ measurements, the tunneling current was maintained constant. The inset of Fig. 1 shows a typical $I_{c}-V$ characteristic obtained on the $\mathrm{Pb}$ wetting layer. We found that, as long as the energy of tunneling electrons, 


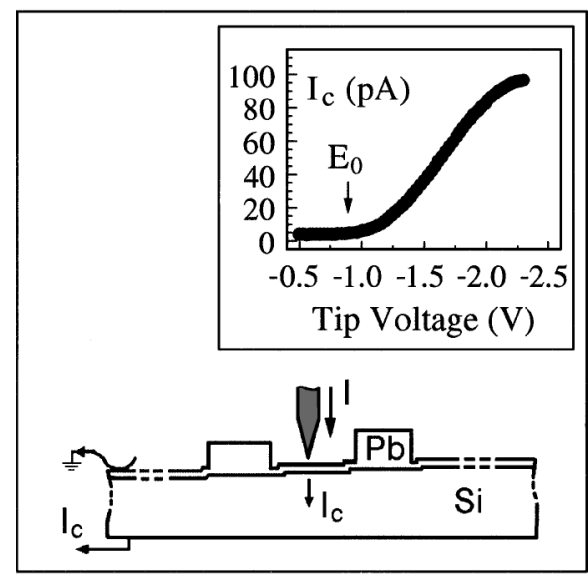

FIG. 1. Schematics of the in situ STM/BEEM experimental setup. Inset: Typical BEEM current-voltage characteristic obtained on the $\mathrm{Pb}$ wetting layer. The threshold $E_{0}=$ $0.85 \mathrm{eV}$ for the electron injection into the bulk corresponds to the $\mathrm{Pb} / \mathrm{Si}(111)$ Schottky barrier height.

$E=e V$, does not exceed the $\mathrm{Pb} / \mathrm{Si}(111)$ Schottky barrier height [18] $\left(E_{0}=0.85 \pm 0.5 \mathrm{eV}\right)$, variations of $I_{c}$ do not exceed the noise level. Obviously, at these energies all the tunneling current is collected from the $2 \mathrm{D} \mathrm{Pb}$ wetting layer. Increasing the energy of the tunneling electrons above $E_{0}$ results in an increase of $I_{c}$, which is initially nearly quadratic and later evolves into a linear dependence on $E$ [19]. This presents a clear evidence that the $\mathrm{Pb}$ wetting layer possesses a metallic conductivity, and, hence, can be used to electrically contact the nanostructures.

The complicated structure of our samples, consisting of objects of different dimensionality, raises a question as to how local the BEEM current is. In order to answer this question, we show in Fig. 2 simultaneously acquired STM and BEEM images (respectively) of our sample. In the STM image, we observe a significant density of surface steps. These steps, however, do not prevent the continuous

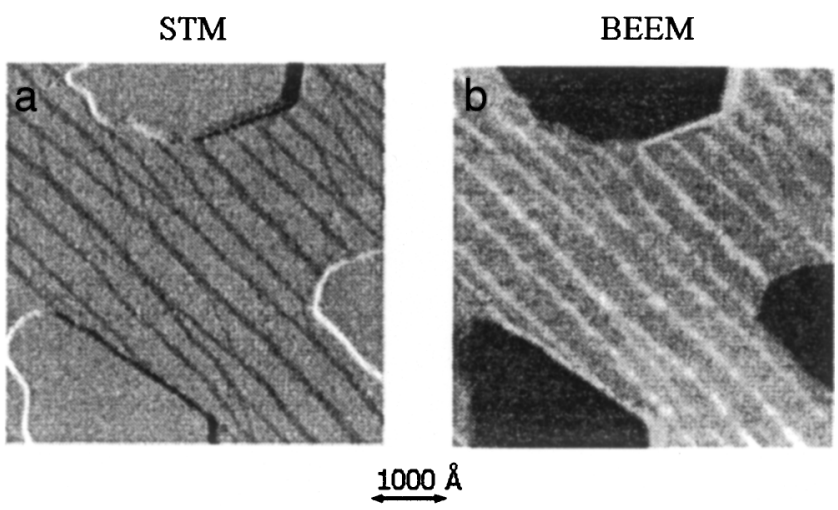

FIG. 2. $5000 \times 5000 \AA$ STM and BEEM images of epitaxial $\mathrm{Pb}(111)$ film on $\mathrm{Si}(111)$ acquired simultaneously at a tip voltage of $-1.5 \mathrm{~V}$. A significantly smaller BEEM current is observed within the borders of the $\mathrm{Pb}$ islands as compared to the surrounding $\mathrm{Pb}$ wetting layer. The gray scale in the BEEM image spans the range between 0 to $12 \mathrm{nA}$. metallic conductivity of the $\mathrm{Pb}$ wetting layer, as the BEEM threshold $E_{0}$ is clearly observed throughout this layer. On top of the wetting layer, we observe $3 \mathrm{D} \mathrm{Pb}$ islands with typical lateral sizes of $2000 \AA$ and thickness varying from 30 to $80 \AA$. Analysis of the STM and BEEM images shows a significant reduction of the electron injection across the $\mathrm{Pb}$ islands as compared to the surrounding $\mathrm{Pb}$ wetting layer [20]. Apparently, most of the electrons, which tunnel into the $\mathrm{Pb}$ islands, thermalize below the Schottky barrier by the time they laterally diffuse into the surrounding wetting layer. As we show below, the electron injection into the semiconductor is laterally localized not only within a single $\mathrm{Pb}$ island but also within a single constant-height region of the wedge-shaped $\mathrm{Pb}$ islands.

In Fig. 3, we show a series of $I_{c}-V$ characteristics obtained from $\mathrm{Pb}$ islands of different thickness. Since the interfacial structure remains intact below $\mathrm{Pb}$ islands [7], the Schottky threshold on the islands is the same as on the wetting layer. However, the shapes of these curves are different. An additional steplike feature appears in all the curves of Fig. 3, at an energy position that is clearly thickness dependent. The position of this steplike feature increases in energy as the film thickness decreases. This clearly suggests that the $I_{c}-V$ features originate from the

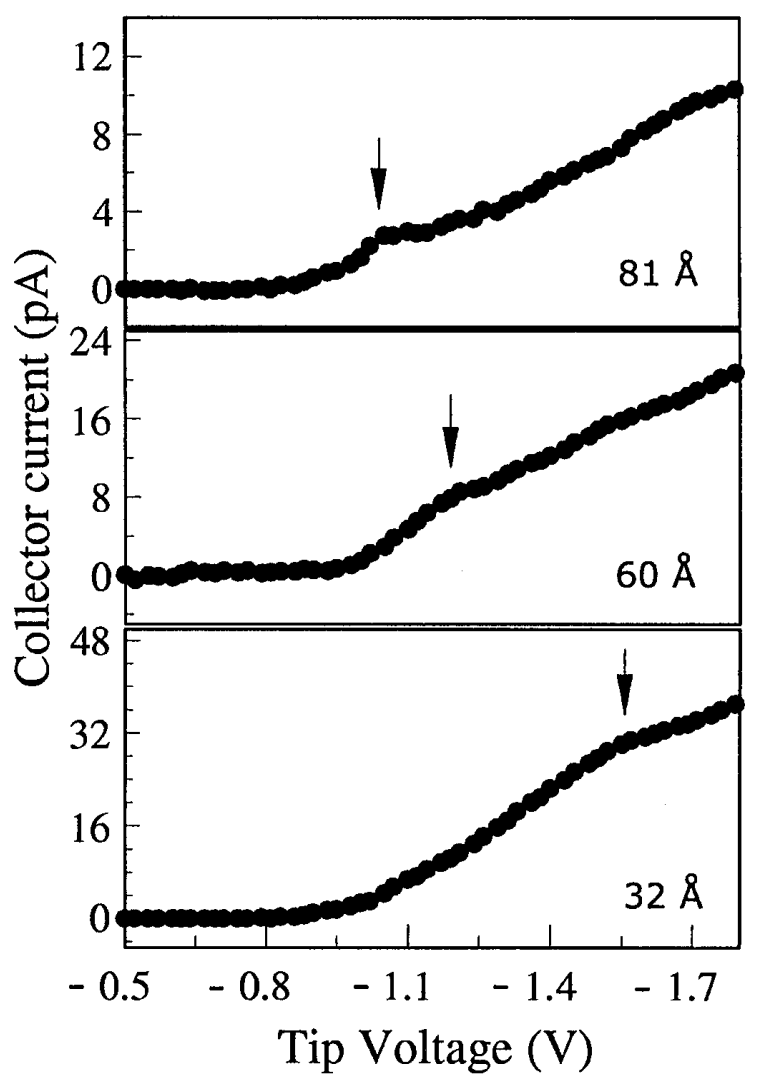

FIG. 3. BEEM current-voltage characteristics obtained on $\mathrm{Pb}$ islands of different thickness. The threshold, $E_{0}$, is the same as on the wetting layer. The additional thickness-dependent features that appear on these curves indicate resonant electron states in $\mathrm{Pb}$ islands. 
resonant electron states in the $\mathrm{Pb}$ islands. A previous study of resonant states in $\mathrm{Pb}$ [4] with scanning tunneling spectroscopy has shown that the electron confinement energy,

$$
\Delta=\frac{\pi \hbar v_{F}}{H}
$$

where $v_{F}$ is the Fermi velocity in $\mathrm{Pb}$, indeed increases as the metal thickness, $H$, decreases. However, unlike in tunneling spectra, where resonant steps repeat with an interval of $\Delta$, the transmission $\left(I_{c}-V\right)$ spectra always contain only one thickness-dependent steplike feature. As we shall show later, this is an inherent property of a three-terminal experiment.

In Fig. 4, we show the typical dependence of the electron injection rate $\left(I_{c} / I\right)$ into the bulk $\mathrm{Si}(111)$ states on the thickness of $\mathrm{Pb}$ film. The data points in Fig. 4 were obtained by translating the STM tip over the top surface of the $\mathrm{Pb}$ wedge at a fixed tunneling current $(10 \mathrm{nA})$ and a fixed voltage $(-1.9 \mathrm{~V})$ and measuring $I_{c}$ for different metal thickness. We observe a continuous decrease of $I_{c}$ with an increase of the metal thickness. Variations of $I_{c}$ with the metal thickness have been attributed to electron attenuation over the mean-free path, $\lambda$, which implies the exponential dependence [21]: $I_{c} \propto \exp (-H / \lambda)$. This model, being valid only in the strong scattering limit, does not account for the alternative regime, i.e., development of standing electron waves in a metallic film $[4,22]$. Standing electron waves in the epitaxial $\mathrm{Pb} / \mathrm{Si}(111)$ films have been directly observed in previous studies of this system $[4,7]$. In those studies, it was shown that the tunneling density of states in $\mathrm{Pb}$ islands exhibits oscillating energy dependence both below and above the $\mathrm{Pb} / \mathrm{Si}$ Schottky barrier. The same experiment conducted at room temperature indicates a ballistic mean-free path of more than $100 \AA$.

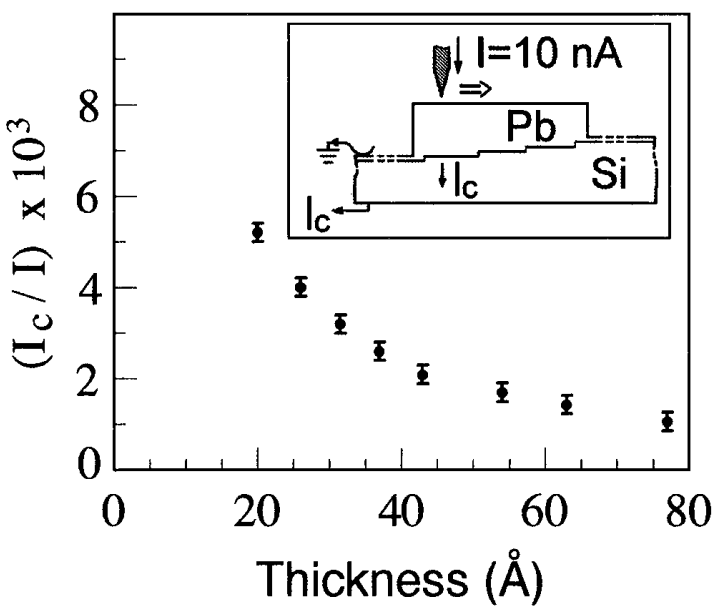

FIG. 4. Dependence of the normalized BEEM current, $I_{c} / I$, on the $\mathrm{Pb}$ thickness. The data points were obtained by translating the STM tip over the top surface on the self-assembled Pb wedge at a fixed tip voltage of $-1.9 \mathrm{~V}$ and a fixed tunneling current of $10 \mathrm{nA}$ (as shown in the inset). The errors are indicated by the vertical bars.
Therefore, the picture of a "particle in a box" seems to be appropriate for the explanation of the thickness-dependent ballistic transport across the $\mathrm{Pb}$ islands.

Consider an electron, of energy $E>E_{0}$, tunneling from a STM tip into a metal QW. The transversal motion of this electron is described by periodic oscillations across the $\mathrm{QW}$ with a frequency $\Omega=v / 2 H$, where $v \approx v_{F}$. Hence, the average charge injection into the semiconductor is given by

$$
Q_{c}=\beta e(\Omega \tau),
$$

where $\beta$ is the interfacial transmission probability, $e$ is the electron charge, and $\tau$ is the bulk electron relaxation time. The product $\beta e$ represents the charge injection per attempt, while $\Omega \tau$ is the integral number of attempts to traverse the metal-semiconductor interface, which is given by the number of electron oscillations inside a $\mathrm{QW}$ that contribute to the interfacial transport. The rest of the charge can spread only through the wetting layer. As shown in a previous study, the low-energy part $\left(E<E_{0}\right)$ of the tunneling current can be neglected at large negative tip voltage [4]. Thus, the ratio of the BEEM current to the tunneling current is given by the ratio between $Q_{c}$ and $e$, and equals

$$
\frac{I_{c}}{I}=\beta(\Omega \tau) \approx \frac{\beta v_{F} \tau}{2 H} .
$$

The inverse proportion of the BEEM current to the QW height, predicted in Eq. (3), can thus explain the decrease of $I_{c}$ with the metal thickness. To show this dependence, we plot $\left(I_{c} / I\right)^{-1}$ as a function of $H$ (Fig. 5). The resulting linear dependence is clearly observed in Fig. 5. Hence, comparing Eqs. (1) and (3), we infer that $I_{c} \propto \Delta$, i.e., the interfacial transport in epitaxial QWs is determined by the electron confinement energy.

Deviations from exponential dependence in thin Au film on $\mathrm{Si}(111)$ were reported earlier [21,23]. However, as bulk resonant states in Au films have never been observed, the

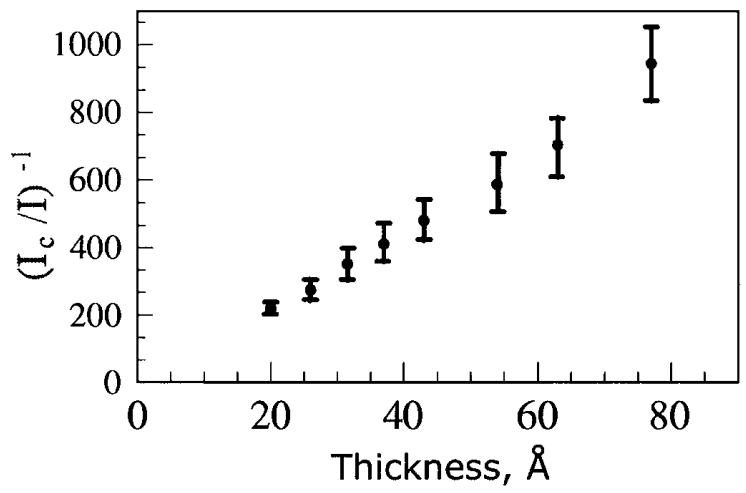

FIG. 5. The inverse of the normalized BEEM current, $\left(I_{c} / I\right)^{-1}$, plotted as a function of the Pb thickness. A proportional dependence is clearly observed. The errors are indicated by the vertical bars. 
scaling predicted by Eq. (3) was not observed as well. Besides, as Au films do not grow in Stranski-Krastanov geometry, direct thickness measurement with STM is not possible. By contrast, the two noticeable features of the $\mathrm{Pb} / \mathrm{Si}(111)$ interface are (i) self-assembly of the wedgeshaped islands, allowing for direct thickness determination, and (ii) electron focusing in the [111] direction due to the Fermi surface anisotropy, enhancing resonant electron states in the $\mathrm{Pb}$ QWs [24]. The combination of these two features allows one to consider a $\mathrm{Pb}$ island a model quantum box system.

As was first recognized by Bardeen [25], in two-terminal transport experiments, such as tunneling into a quantum box, the scaling effect cannot be manifested. Transport in these systems can be described as $M^{2} \rho$, where $M$ is the tunneling matrix element, and $\rho$ is the density of states. Because of $M \propto \psi \propto 1 / \sqrt{H}$, where $\psi$ is the electron wave function in a quantum box, and $\rho \propto H$, the scaling effect always vanishes. By contrast, in three-terminal geometry, the flux across a quantum box can be described as $M^{2} N$, where $N=(I / e) \tau$ is the total population number of resonant states in energy window $E_{0}<E<e V$, and the matrix element $M$ couples $\psi$ with the collector. It now becomes clear why only single $I_{c}-V$ steps are manifested in the transmission spectra. As the opening of additional resonant channels does not change $N$, the higher order resonant states in these spectra are not visible.

Thus, in the quantum mechanical picture, a threeterminal experiment with a quantum box allows direct measurement of $|\psi|^{2}$. It seems remarkable that such measurement is facilitated by electron-phonon interaction, which represents the main mechanism of bulk relaxation in our experiment.

In conclusion, confinement-enhanced electron transport across an epitaxial metal-semiconductor interface was observed with the combination of STM and BEEM. Using modern methods of nanofabrication and in situ characterization, we have designed a three-terminal experiment with a quantum box and experimentally observed quantum scaling of its transmittance. Utilization of a quantum box in a three-terminal geometry may possibly open a way to development of nanoscale quantum transistors.

We thank K. A. Matveev, D. M. Chen, I. Shalish, and R.E. Martinez for fruitful discussions. We acknowledge support from the NSF through Harvard MRSEC (DMR 98-09363) and DARPA (DAAD 19-99-1-0158).

[1] M. Jalochowski, M. Hoffman, and E. Bauer, Phys. Rev. Lett. 76, 4227 (1996).

[2] X. Tong, K. Horikoshi, and S. Hasegawa, Phys. Rev. B 60, 5653 (1999).

[3] D. A. Evans et al., Phys. Rev. Lett. 70, 3483 (1993).

[4] I. B. Altfeder, K. A. Matveev, and D. M. Chen, Phys. Rev. Lett. 78, 2815 (1997).

[5] J. J. Paggel, T. Miller, and T. C. Chiang, Science 283, 1709 (1999).

[6] H. Hong et al., Phys. Rev. Lett. 68, 507 (1992).

[7] I. B. Altfeder, D. M. Chen, and K. A. Matveev, Phys. Rev. Lett. 80, 4895 (1998).

[8] G. Binnig et al., Phys. Rev. Lett. 50, 120 (1983).

[9] W. J. Kaiser and L. D. Bell, Phys. Rev. Lett. 60, 1406 (1988).

[10] M. Prietsch, Phys. Rep. 253, 164 (1995).

[11] L. D. Bell and W. J. Kaiser, Annu. Rev. Mater. Sci. 26, 189 (1996).

[12] L. D. Bell and V. Narayanamurti, Curr. Opin. Solid State Mater. Sci. 3, 38 (1998).

[13] M. Prietsch and R. Ludeke, Phys. Rev. Lett. 66, 2511 (1991).

[14] T. Sajoto et al., Phys. Rev. Lett. 74, 3427 (1995).

[15] M. E. Rubin et al., Phys. Rev. Lett. 77, 5268 (1996).

[16] T. Meyer et al., Phys. Rev. Lett. 85, 1520 (2000).

[17] M. Jalochowski and E. Bauer, J. Appl. Phys. 63, 4502 (1988).

[18] J. A. Carlisle, T. Miller, and T.-C. Chiang, Phys. Rev. B 45, 3400 (1992).

[19] Fitting the data to well-known BEEM theories [10,11] yields $E_{0}=0.83 \mathrm{eV}$.

[20] A signal enhancement at the edges of the $\mathrm{Pb}$ islands and at the surface steps in the BEEM image of Fig. 2 is due to transient effects in the STM feedback loop.

[21] M. K. Weilmeier, W. H. Rippard, and R. A. Buhrmann, Phys. Rev. B 59, R2521 (1999).

[22] R. C. Jaklevic et al., Phys. Rev. Lett. 26, 88 (1971).

[23] L. D. Bell, Phys. Rev. Lett. 77, 3893 (1996).

[24] J. R. Anderson and A. V. Gold, Phys. Rev. 139, A1459 (1965).

[25] J. Bardeen, Phys. Rev. Lett. 6, 57 (1961). 